\title{
Methods Calculating the Slab Radiance Factor
}

\author{
Vladimir Budak ${ }^{10000-0003-4750-0160]}$, Dmitry Efremenko ${ }^{20000-0002-7449-5072]}$ \\ ${ }^{1}$ National Research University "Moscow Power Engineering institute”, Moscow, 111250 Russia \\ budakvp@gmail.com \\ ${ }^{2}$ Remote Sensing Technology institute (IMF), German Aerospace Center (DLR), Oberpfaffenhofen, \\ 82234 Wessling, Germany \\ dmitry.efremenkoddlr.de
}

\begin{abstract}
One of the most critical problems of realistic visualization of the real-world objects is physically adequate modeling of their reflection of light. Reflection of light by objects occurs both from the surface and the bulk of matter (scattering). Accounting for the light reflection from the surface of objects was solved almost a century ago based on its representation as a Fresnel randomly rough surface. Scattering by a bulk of matter is the subject of radiation transfer theory, which has only recently received its known completion in the form of discrete transfer theory. Strict analytical methods for solving the radiation transport equation (RTE) are often not highly effective for calculating the radiance factor. For a long time, in the absence of effective numerical methods for solving problems and the unavailability of high-speed computers for practical calculations, approximate methods for solving RTE were developed. However, their accuracy and applicability limits were poorly defined. The discrete transfer theory allowed us to evaluate the existing approximate methods for solving the UPI, their accuracy, and the efficiency of application for calculating the radiance factor. It is shown that the most effective method is the method of synthetic iterations. The method is based on the selection of the solution anisotropic part based on a small-angle approximation of the RTE solution. The solution regular part can be calculated by any approximation. Then a simple iteration from the complete solution is performed to refine the angular distribution of the radiance factor.
\end{abstract}

Keywords: Radiance Factor, Reflectance, Radiative Transfer, Synthetic Iteration, Discrete Ordinates.

\section{Radiance factor}

One of the most pressing problems of computer graphics (CG) today is the mathematical model of reflection by objects of the real world [1, 2]. The concept of light reflection was introduced to optics by the first physicist on earth, Abu Ali al-Haysam [3], in the XI century.

Copyright (C) 2020 for this paper by its authors. Use permitted under Creative Commons License Attribution 4.0 International (CC BY 4.0). 
It is difficult to list all the names in a short article that the theory of reflection owes its development over 1000 years. Common reflection characteristics were introduced by $\mathrm{C}$. Fabry [4]. A complete reflection characteristic is the surface radiance factor, which, according to the International lighting vocabulary [5], is determined as the ratio of the radiance of the surface element in the given direction to that of the perfect reflecting or transmitting diffuser identically irradiated and viewed.

Since the lighting conditions are not predefined, the radiance factors for diffuse and directional lighting should be distinguished. Let us consider the radiance $L\left(\mathbf{r}, \hat{\mathbf{I}}^{\prime}\right)$ at the point defined by the radius-vector $\mathbf{r}$ and propagating along the direction $\hat{\mathbf{l}}^{\prime}$. Then, the radiance factor $\rho\left(\mathbf{r} ; \hat{\mathbf{l}}, \hat{\mathbf{l}}^{\prime}\right)$ in the viewing direction $\hat{\mathbf{l}}$ due to the directed illumination of the studied surface area at the point $\mathbf{r}$ in the direction $\hat{\mathbf{I}}^{\prime}$ is defined as follows:

$$
\rho\left(\boldsymbol{r} ; \hat{\boldsymbol{l}}, \hat{\boldsymbol{l}}^{\prime}\right)=\pi \frac{L\left(\boldsymbol{r}, \hat{\boldsymbol{l}}^{\prime}\right)}{E(\boldsymbol{r})(\widehat{N}, \hat{l})},
$$

where $L\left(\mathbf{r}, \hat{\mathbf{I}}^{\prime}\right)$ is a visible surface radiance, $E(\mathbf{r})$ is normal irradiance from incident radiation, $\hat{\mathbf{N}}$ is the surface normal at the point $\mathbf{r}$.

It is straight forward to see that according to (1) the radiance factor is the same as the definition of bidirectional reflectance distribution function (BRDF). However, according to [4], the term radiance factor is applicable for both reflected and transmitted radiation. Accordingly, if a thin horizontal layer is irradiated from above $L_{\downarrow}\left(\mathbf{r}, \hat{\mathbf{I}}^{\prime}\right)$ and below $L_{\uparrow}\left(\mathbf{r}, \hat{\mathbf{I}}^{\prime}\right)$, the radiance $L(\mathbf{r}, \hat{\mathbf{l}})$ of the radiation coming out of the layer can be represented as:

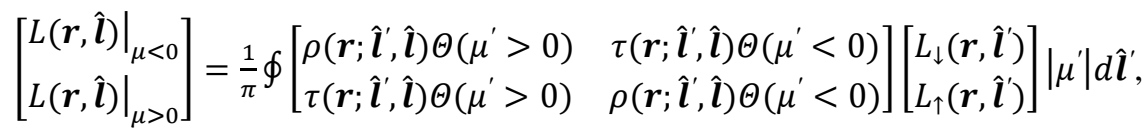

where $\tau\left(\mathbf{r} ; \hat{\mathbf{l}}^{\prime}, \hat{\mathbf{l}}\right)$ is the radiance factor by transmission, $\mu^{\prime}=\left(\hat{\mathbf{N}}, \hat{\mathbf{I}}^{\prime}\right), \mu=(\hat{\mathbf{N}}, \hat{\mathbf{l}})$. The $\Theta$ function is equal to 1 if the argument value is true, and 0 if it is false.

Thus, in the general case, the slab is characterized by a matrix of radiance factor. For radiance factors, the principle of reciprocity is observed: transmission and reflection from top to bottom and from bottom to top are equal.

In [6], a model of slab reflection was proposed, which determined all further efforts in this direction. The model [6] is based on the representation of the reflection process as the sum of two processes: a reflection from the Fresnel randomly rough surface (RRS)of the interface between media and light scattering by the thickness of substances. The development of the RRS reflection model has been very productive. In the article [7], the primary relations of reflection from the RRS were formulated, and subsequent works were mainly its refinements. 
The problem of reflection (scattering) of light by the entire volume of the layer is reduced to a boundary value problem for the radiation transfer equation (RTE), which turned out to be a mathematically more complex problem, practical algorithms for solving which in fact only by the end of the XX century. In [8,9], a complete, analytically rigorous model of radiation reflected by a layer was proposed. With all the advantages of this model, it requires setting environment such characteristics that are not clear to the average user of the software.

However, before precise methods for solving RTE were developed, approximate methods were developed that were limited in their scope. They were commonly referred to as engineering methods since they were often used in the design of various devices. These methods were often developed separately, based on some narrow assumptions about the properties of the medium and the light field inside. From the present time, when a strict analytical solution of the RTE is available, it is of considerable interest to assess the accuracy and applicability limits of these engineering approximations. It will allow us to determine their suitability for use in CG algorithms.

\section{Discrete radiative transfer theory}

We start our analysis with the boundary value problem formulation for the radiance field $L(\tau, \hat{\mathbf{I}})$ in the case of a homogeneous slab of the optical thickness $\tau_{0}$. Here $\tau$ is the vertical coordinate measured in dimensionless optical thickness and is zero at the layer top, while $\hat{\mathbf{l}}=\{\mu, \varphi\}$ is the direction given in spherical coordinates by the cosine of polar angle $\mu$ and the azimuthal angle $\varphi$. The slab is illuminated by a mono-directional plane source at the top of the layer with $\hat{\mathbf{I}}_{0}=\left\{\mu_{0}, \varphi_{0}\right\}$ being the direction of incidence, $\mu_{0}$ the cosine of the polar incident angle, while $\varphi_{0}=0$. The boundary value problem for $L(\tau, \hat{\mathbf{I}}) \operatorname{reads}$ as follows [10]:

$$
\left\{\begin{array}{c}
\mu \frac{\partial L(\tau, \hat{\boldsymbol{l}})}{\partial \tau}+L(\tau, \hat{\boldsymbol{l}})=\frac{\Lambda}{4 \pi} \oint L\left(\tau, \hat{\boldsymbol{l}}^{\prime}\right) x\left(\hat{\boldsymbol{l}}, \hat{\boldsymbol{l}}^{\prime}\right) d \hat{\boldsymbol{l}}^{\prime}, \\
\left.L(\tau, \hat{\boldsymbol{l}})\right|_{\tau=0, \mu>0}=\delta\left(\hat{\boldsymbol{l}}-\hat{\boldsymbol{l}}_{0}\right),\left.\quad L(\tau, \hat{\boldsymbol{l}})\right|_{\tau=\tau_{0}, \mu<0}=0,
\end{array}\right.
$$

where $x$ is the single scattering phase function.

Direct numerical solution of Eq. (3) is impossible due to singularities in $L(\tau, \hat{\mathbf{l}})$ (i.e. the delta-function) coming from the upper boundary conditions. To overcome this problem, the total radiation is expressed as a sum of the direct radiation and the diffuse radiation [11]. Note that the first (singular) part can be evaluated analytically, while the diffuse part can be found numerically. In practice, this approach is not efficient when the single scattering phase function is forward peaked (e.g. due to large particles compared to the wavelength of 
the light [12]), for which several hundreds of Legendre expansion coefficients have to be taken into account. For the sake of numerical efficiency, in addition to the singular part, the so called small-angle part of the radiance is treated analytically, while the remaining part of the radiance field is smooth. This framework is based on the following representation of the radiance field [13]:

$$
L(\tau, \hat{\boldsymbol{l}})=L_{a}(\tau, \hat{\boldsymbol{l}})+\tilde{L}(\tau, \hat{\boldsymbol{l}}),
$$

where $L_{a}(\tau, \hat{\mathbf{l}})$ is the anisotropic part which incorporates all singularities in the angular domain, while $\mathcal{L}(\tau, \hat{\mathbf{l}})$ is the regular part of the solution. As shown in [13], substituting Eq. (4) into Eq. (3) gives a new boundary problem with an additional source term. The numerical solution based on the discrete ordinate method involves the following steps:

- Fourier cosine expansion of the radiance and phase functions;

- discretization of the radiance field in the angular domain by introducing a set of Gaussian points $\left\{\mu_{i}^{ \pm}\right\}$and weights $\left\{w_{i}\right\}, i=1, \ldots, N$, where $N$ is the number of discrete ordinates per hemisphere, the upper index ' + ' refers to the downwelling radiance, while '-' corresponds to the upwelling radiance.

- replacing the scattering integral with a finite sum [14] in the discrete ordinate space;

- transformation of the radiative transfer equation into the differential matrix equation with respect to column vectors consisting of the radiance values at Gaussian nodes.

After performing these steps, the original problem is transformed into the following equation:

$$
\frac{d \vec{C}(\tau)}{d \tau}=-\overleftrightarrow{B} \vec{C}(\tau)+\overleftrightarrow{M}^{-1} \vec{\Delta}(\tau)
$$

where $\vec{C}(\tau) \equiv\left[\vec{C}_{-}(\tau), \quad \vec{C}_{+}(\tau)\right]^{T}, \vec{C}_{ \pm}(\tau) \equiv\left\{L^{m}\left(\tau, \mu_{i}^{ \pm}\right)\right\}_{i=\overline{1, N}}$, B is the layer matrix, $\vec{\Delta}$ is the source term, while $\stackrel{\grave{M}}{\mathrm{M}}=\operatorname{diag}\left(\mu_{i}^{-}, \mu_{i}^{+}\right)$. Expressions for $\mathrm{B}$ and $\vec{\Delta}$ are given in [13]. The general solution of the inhomogeneous equation (5) is given as the sum of the particular solution of the inhomogeneous equation and general solution of the homogeneous equation:

$$
-\vec{C}(0)+\overleftrightarrow{P}\left(0, \tau_{0}\right) \vec{C}\left(\tau_{0}\right)=\int_{0}^{\tau_{0}} \stackrel{\leftrightarrow}{P}(0, \tau) \overleftrightarrow{M}^{-1} \vec{\Delta}\left(\tau, \mu_{0}\right) d \tau
$$

where

$$
\overleftrightarrow{P}(t, \tau) \equiv e^{\overleftrightarrow{B}(\tau-t)}
$$

is the solution to the homogeneous equation. $\overleftrightarrow{P}(t, \tau)$ is referred to as the propagator and relates the radiance fields at spatial coordinates $t$ and $\tau$. That means, if a radiance field at $\tau$ is known, it can be 'propagated' to the point $t$ by applying the propagator (7). However, our original problem (3) is a two-point boundary value problem, i.e. at each boundary only a half of boundary conditions (for one hemisphere) is provided. Therefore, solution (6) 
based on the propagators cannot be readily applied.

To solve the two-point boundary value problem, the eigenvalue decomposition of the matrix exponential in Eq. (7) should be performed. Then, applying the so called scaling transformation [15] and taking into account the boundary conditions, a robust solution of Eq. (6) can be obtained, which can be written in the form of scatters (see detailed derivations in [13]):

$$
\left[\begin{array}{l}
\vec{C}_{-}(0) \\
\vec{C}_{+}\left(\tau_{0}\right)
\end{array}\right]=\left[\begin{array}{l}
\vec{F}_{-} \\
\vec{F}_{+}
\end{array}\right]+\left[\begin{array}{ll}
\overleftrightarrow{R}_{-} & \overleftrightarrow{T}_{-} \\
\overleftrightarrow{T}_{+} & \overleftrightarrow{R}_{+}
\end{array}\right]\left[\begin{array}{l}
\vec{C}_{+}(0) \\
\vec{C}_{-}\left(\tau_{0}\right)
\end{array}\right]
$$

where $\vec{F}_{ \pm}$are the source vectors, which correspond to the intrinsic radiation of the layer, while $\overleftrightarrow{R}$ and $\overleftrightarrow{T}$ are the reflection and transmission matrices incorporating the discrete values of reflection and transmission coefficients, respectively. Note that $\vec{F}_{ \pm}, \overleftrightarrow{R}$ and $\overleftrightarrow{T}$ are expressed in terms of eigenvectors of the layer matrix (explicit expressions can be found, e.g. in [16]). Obviously, the column-vectors $\vec{C}_{+}(0), \vec{C}_{-}\left(\tau_{0}\right)$ in Eq. (8) correspond to the radiances, sticking the slab, and are defined by the boundary conditions. The columnvectors $\vec{C}_{-}(0), \vec{C}_{+}\left(\tau_{0}\right)$ correspond to the transmitted and reflected radiances. Thus, Eq. (8) relates the incoming and outgoing radiances and, thus, can be considered as a generalization of the radiance factor (2)

Equation (8) can be transformed back into the propagator-like form suited for one-point boundary value problem:

$$
\vec{C}\left(\tau_{0}\right)=\left[\begin{array}{cc}
\overleftrightarrow{T}_{+}-\overleftrightarrow{R}_{-} \overleftrightarrow{T}_{-}^{-1} \overleftrightarrow{R}_{+} & \overleftrightarrow{R}_{-} \overleftrightarrow{T}_{-}^{-1} \\
-\overleftrightarrow{T}_{-}^{-1} \overleftrightarrow{R}_{+} & \overleftrightarrow{T}_{-}^{-1}
\end{array}\right] \vec{C}(0)+\overleftrightarrow{F}
$$

In [17] such kind of transformation was referred to as the stellar product. The entries of scatter matrices satisfy Riccati equation, which correspond to the one-point boundary value problem. Note that Eqs. (8)-(9) are the strict analytical solution to Eq. (3) in the form of brightness coefficient matrices.

Two comments are in order. First, following Kolmogorov [18], the physical laws are established by discrete measurements, and then formulated in term in a continuous setting. One can argue that nowadays it is more productive and efficient to formulate physical laws (at least in light engineering) directly in a discrete form. Secondly, the accuracy of the solution is determined by the number of ordinates used. The use of many ordinates places high demands on computer power. As the solution is based on the matrix multiplication, the matrix inversion and the eigen decomposition, the computational time increases faster as $N^{2}$. Therefore, engineering applications require high-performance approximate solution techniques, which would be less demanding on computational power. 


\section{Radiance field inside a slab}

After the solution of the discrete RTE, taking into account the boundary conditions, the two-points boundary value problem is transformed into two one-point boundary value problems (with initial conditions) that allows using each of them to determine the radiance field inside the slab [19]:

- through the field on the upper boundary

$$
\overleftrightarrow{U}^{-1} \vec{C}(\tau)=e^{-\overleftrightarrow{\Gamma} \tau} \overleftrightarrow{U}^{-1} \vec{C}(0)+e^{-\overleftrightarrow{\Gamma} \tau} \int_{0}^{\tau} e^{\overleftrightarrow{T} t} \overleftrightarrow{U}^{-1} \overleftrightarrow{M}^{-1} \vec{\Delta}\left(t, \mu_{0}\right) d t,
$$

- through the field on the lower boundary

$$
\overleftrightarrow{U}^{-1} \vec{C}(\tau)=e^{\vec{\Gamma}\left(\tau_{0}-\tau\right)} \overleftrightarrow{U}^{-1} \vec{C}\left(\tau_{0}\right)-e^{-\vec{\Gamma} \tau} \int_{\tau}^{\tau_{0}} e^{\vec{T} t} \overleftrightarrow{U}^{-1} \overleftrightarrow{M}^{-1} \vec{\Delta}\left(t, \mu_{0}\right) d t
$$

Note that the same function is on the left side of both formulae (10) - (11), and the expressions on the right sides are different ones. In this case, expression (10) contains only positive exponents for the upper hemisphere of sighting directions, and expression (11) contain the same for the lower one. Since left sides are equal, then right sides are also equal. That allows us to take the expression for the lower hemisphere from (10), and for the upper hemisphere from (11):

$$
\begin{aligned}
& \overleftrightarrow{U}^{-1} \vec{C}=\left[\begin{array}{c}
e^{\vec{\Gamma}_{-}\left(\tau_{0}-\tau\right)} \overleftrightarrow{\mathrm{u}}_{11} \vec{C}_{-}\left(\tau_{0}\right)+e^{\vec{T}_{-}\left(\tau_{0}-\tau\right)} \overleftrightarrow{\mathrm{u}}_{12} \vec{C}_{+}\left(\tau_{0}\right) \\
e^{-\vec{\Gamma}_{+} \tau \overleftrightarrow{\mathrm{u}}_{21} \overrightarrow{\mathrm{C}}_{-}(0)}
\end{array}\right]+ \\
& e^{-\vec{\Gamma} \tau}\left[\begin{array}{c}
-\left(\int_{\tau}^{\tau_{0}} e^{\overleftrightarrow{\Gamma} t} \overleftrightarrow{U}^{-1} \overleftrightarrow{M}^{-1} \vec{\Delta}\left(t, \mu_{0}\right) d t\right)_{-} \\
\left(\int_{0}^{\tau} e^{\vec{\Gamma} t} \overleftrightarrow{U}^{-1} \overleftrightarrow{M}^{-1} \vec{\Delta}\left(t, \mu_{0}\right) d t\right)_{+}
\end{array}\right] .
\end{aligned}
$$

The obtained expression contains only negative exponents. Therefore, the radiance field calculation does not present any problems for the arbitrary slab optical depth. Substituting the expression for the source function in (6) and calculating the corresponding integrals in (12), we get the resultant expression for the radiance field inside the slab:

$$
\overleftrightarrow{U}^{-1} \vec{C}(\tau)=\left[\begin{array}{cc}
e^{\vec{T}_{-}}\left(\tau_{0}-\tau\right) & 0 \\
0 & e^{-\vec{\Gamma}_{+} \tau}
\end{array}\right] \vec{G}\left(\tau_{0}\right)+\vec{F}(\tau)
$$

where

$$
\begin{gathered}
\vec{F}(\tau) \equiv \sum_{k=m}^{K} \frac{2 k+1}{4 \pi} q_{k} \operatorname{diag}\left(\frac{d_{k} e^{-d_{k} \frac{\tau}{\mu_{0}}}}{\gamma_{i} \mu_{0}-d_{k}}-\frac{e^{-\frac{\tau}{\mu_{0}}}}{\gamma_{i} \mu_{0}-1}\right) \overleftrightarrow{U^{-1}}\left(\overleftrightarrow{1}-\mu_{0} \overleftrightarrow{M}^{-1}\right) \vec{Q}_{k}^{m}, \\
\vec{G}\left(\tau_{0}\right) \equiv\left[\begin{array}{c}
\overleftrightarrow{u}_{11} \vec{C}_{-}\left(\tau_{0}\right)+\overleftrightarrow{u}_{12} \vec{C}_{+}\left(\tau_{0}\right) \\
\overleftrightarrow{u}_{21} \vec{C}_{-}(0)
\end{array}\right]-\left[\begin{array}{c}
\left(\vec{F}\left(\tau_{0}\right)\right)_{-} \\
(\vec{F}(0))_{+}
\end{array}\right] .
\end{gathered}
$$


The radiance angular distributions at some points of the turbid medium are presented in Fig. 1 as polar diagrams.

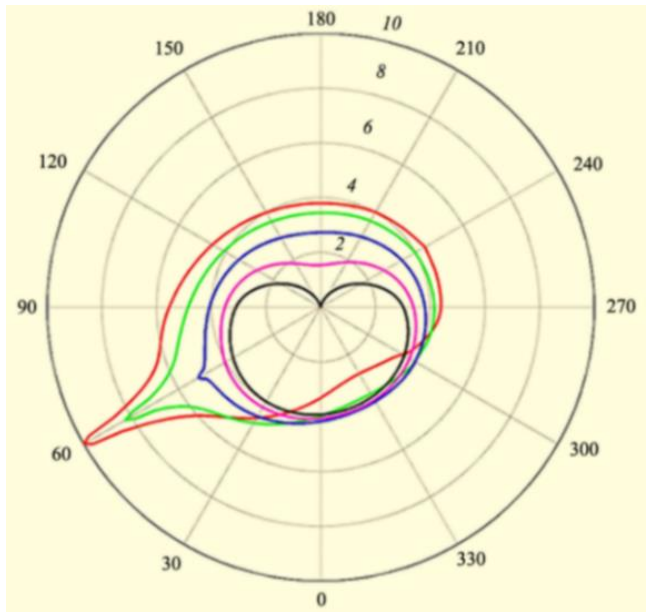

Fig. 1. Normalized radiance angular distribution inside the slab: aerosol, $\mathrm{t}_{0}=10, \mathrm{~L}=1.0, \mathrm{q}_{0}=60^{\circ}$. Red line $-\mathrm{t}=0.01 \mathrm{t}_{0}$, green $-\mathrm{t}=0.25 \mathrm{t}_{0}$, blue $-\mathrm{t}=0.50 \mathrm{t}_{0}$, magenta $-\mathrm{t}=0.75 \mathrm{t}_{0}$, black $-\mathrm{t}=0.90 \mathrm{t}_{0}$.

\section{Analysis of approximate methods}

Most of the approximate solution techniques were derived in the XX century, when there was a lack of computational power even to perform summation across thousands of Legendre expansion coefficients. Even the methods outlined below are not sufficiently accurate, they are several orders of magnitude faster than the rigorous discrete ordinate solution. Nevertheless, as we will see later, the ideas behind these approximate techniques can be reused in the framework of state-of-the-art computational codes for engineering applications.

\subsection{Two-Stream Approximation}

A natural approximation based on the theory outlined in Section 2 is the two-stream approximation. It can be derived by setting $N$ to 1 . Thus, we have one discrete ordinate per hemisphere. Physically the problem (3) is reduced to the case in which we are interested only in irradiances of upwelling and dowelling radiation. Despite that fact that the radiance distribution across the polar hemispheres is not considered, the radiant energy is preserved.

Note that such an approximation was formulated by Schuster [20] and Schwarzschild [21] in the framework of astrophysical radiative transfer. Mathematically, the feature of the two-stream approximation is that the eigenvalue problem can be solved analytically, and the solution can be expressed in a closed form, thereby yielding high computational speed. 


\subsection{Diffuse approximation}

We present all functions in the RTE in the boundary value problem (3) as a spherical function expansion for the case $\mathrm{P}_{1}$ of the spherical harmonics method:

$$
\begin{gathered}
L_{r}(\tau, \hat{\mathbf{l}})=C_{00}(\tau) \mathrm{Y}_{0}^{0}(\hat{\mathbf{l}})+\sum_{m=-1}^{1} C_{1 m}(\tau) \mathrm{Y}_{1}^{m}(\hat{\mathbf{l}})=\frac{1}{4 \pi}\left(E_{0}(\tau)+3 \mathcal{E}(\tau) \hat{\mathbf{l}}\right), \\
\Delta(\tau, \hat{\mathbf{l}})=s_{00}(\tau) \mathrm{Y}_{0}^{0}(\hat{\mathbf{l}})+\sum_{m=-1}^{1} s_{1 m}(\tau) \mathrm{Y}_{1}^{m}(\hat{\mathbf{l}}) \equiv \frac{1}{4 \pi}\left(s_{0}(\tau)+3 \mathbf{s}(\tau) \hat{\mathbf{l}}\right), \\
x(\hat{\mathbf{l}} \cdot \hat{\mathbf{l}})=\sum_{l=0}^{\infty} \frac{21+1}{4 \pi} x_{l} \mathrm{P}_{l}\left(\hat{\mathbf{l}}^{\prime} \cdot \hat{\mathbf{l}}\right),
\end{gathered}
$$

where $E_{0}(\tau) \equiv \oint L_{r}(\tau, \hat{\boldsymbol{l}}) d \hat{\boldsymbol{l}}, \quad E(\tau) \equiv \oint L_{r}(\tau, \hat{\boldsymbol{l}}) \hat{\boldsymbol{l}} d \hat{\boldsymbol{l}}$ are spatial irradiance and light vector, respectively, and similarly $s_{0}(\tau), \quad \mathbf{s}(\tau)$ for the source function; $\mathrm{Y}_{k}^{m}(\hat{\mathbf{I}})=\sqrt{\frac{2 k+1}{4 \pi}} \sqrt{\frac{(l-n) !}{(l+n) !}} \mathrm{P}_{l}^{n}(\mu) \mathrm{e}^{i m \varphi}, \mathrm{P}_{l}^{n}(\mu)$ are the associated Legendre polynomials. $C_{k m}, s_{k m}$ are coefficients of radiance expansion and source functions by spherical functions.

After substituting (16)-(18) in RTE, we consistently integrate the resulting expression by the full solid angle and with the weight $\hat{\imath}$. Taking into account the orthogonality of spherical functions we obtain a connected system of two equations [22]:

$$
\left\{\begin{array}{l}
\varepsilon(1-\Lambda) E_{0}(\tau)+\nabla \mathcal{E}(\tau)=s_{0}(\tau), \\
\frac{1}{3} \nabla E_{0}(\tau)+\varepsilon\left(1-\Lambda x_{1}\right) \mathcal{E}(\tau)=\mathbf{s}(\tau),
\end{array}\right.
$$

that corresponds to the $\mathrm{P}_{1}$-approximation of the spherical harmonics method [22].

Under the assumption that the medium is homogeneous $\mathrm{e}=$ const, we can calculate the divergence from the second equation of the system (19), express $\nabla \mathcal{E}(\tau)$ from the first and substitute it into the second, which finally leads to the equation [22]:

$$
\frac{1}{a^{2}} \Delta E_{0}(\tau)-E_{0}(\tau)=\psi(\tau),
$$

where $a=\varepsilon \sqrt{(1-\Lambda)\left(1-\Lambda x_{1}\right)}, \psi(\tau) \equiv-\frac{s_{0}(\tau)}{3 \varepsilon(1-\Lambda)}+\frac{\nabla \mathbf{s}(\tau)}{3 a^{2}}$ is the source function.

Equation (20) admits an analytical solution. 


\subsection{Invariant imbedding and matrix operator method}

The invariant imbedding method was proposed by V. A. Ambartsumian in the first half of the $20^{\text {th }}$ century [23]. This method is based on the fact that the addition to the semi-infinite medium of a layer with an arbitrary thickness and the same optical properties does not change the reflected light intensity. As the layer can be of arbitrary thickness, we add an infinitely thin layer, so that in this layer the multiple scattering collisions can be neglected. The resulting equation is formulated for the reflection function. Note, that here we deal with the one-point boundary value problem. In the discrete ordinate setting, the Ambartsumian equation is transformed into matrix Riccati equation [24].

A consequence of the invariant imbedding principle is the so-called matrix operator method. Considering Eq. (8) for two adjacent layers, a similar equation can be derived for an efficient layer, which has the same optical properties as the system of two-layers [13]. Note that this principle can be used for the adding-doubling algorithm, in which the reflection and transmission matrices are found for a very thin layer using a certain approximation, and then by multi-fold doubling the corresponding entries for the desired optical thickness can be obtained.

\subsection{Method of iterations}

The radiation transfer equation was first formulated by Chwolson [25] and Lommel [26] in photometry for calculating the transmission and reflection of milk glasses. The equation was expressed in an integral form, and the solution was proposed by the iteration method.

In the method of iterations, the diffuse radiance can be expressed as follows [27]:

$$
L=\sum_{s=0}^{N_{s}} L_{s}
$$

where $L_{s}$ refers to the $s$-fold scattered radiance, while $N_{s}$ the maximum scattering order considered. The expansion (21) is called Neumann series, and the computational technique based on Eq. (21) is called successive order of scattering (SOS). The computations of $L_{s}$ can be organized iteratively. Let us consider the radiative transfer equation from Eq. (3),

$$
\mu \frac{\partial L(\tau, \hat{\mathbf{l}})}{\partial \tau}+L(\tau, \hat{\mathbf{l}})=J(\tau, \hat{\mathbf{l}})
$$

in which the multiple scattering term is denoted by the source function $J(\tau, \hat{\mathbf{l}})$, i.e.

$$
J\left(\tau, \hat{\boldsymbol{l}}^{\prime}\right)=\frac{\Lambda}{4 \pi} \oint L\left(\tau, \hat{\boldsymbol{l}}^{\prime}\right) x\left(\hat{\boldsymbol{l}}, \hat{\boldsymbol{l}}^{\prime}\right) d \hat{\boldsymbol{l}}^{\prime} .
$$

At the zero-th iteration we set

$$
L_{0}(\tau, \hat{\mathbf{l}})=e^{-\tau / \mu_{0}} \delta\left(\hat{\mathbf{l}}-\hat{\mathbf{l}}_{0}\right)
$$


and evaluate $J_{0}\left(\tau, \hat{\mathbf{I}}^{\prime}\right)$ according to Eq. (23). Now the right hand side of Eq. (22) is known and Eq. (22) can be integrated, yielding for the diffuse downwelling and upwelling radiation

$$
L_{s}(\tau, \hat{\mathbf{l}})=\int_{0}^{\tau} J_{s}\left(\tau^{\prime}, \hat{\mathbf{l}}\right) e^{-\frac{\tau-\tau^{\prime}}{\mu}} \frac{d \tau^{\prime}}{\mu}
$$

and

$$
L_{s}(\tau, \hat{\mathbf{l}})=L_{s}\left(\tau_{0}, \hat{\mathbf{l}}\right) \mathrm{e}^{-\frac{\tau_{L}-\tau}{|\mu|}}+\int_{\tau}^{\tau_{L}} J_{s}\left(\tau^{\prime}, \hat{\mathbf{l}}\right) \mathrm{e}^{-\frac{\tau^{\prime}-\tau}{|\mu|}} \frac{d \tau^{\prime}}{|\mu|} .
$$

respectively. Then $L_{s}(\tau, \hat{\mathbf{l}})$ is used to find $J_{s+1}\left(\tau, \hat{\mathbf{I}}^{\prime}\right)$ from Eq. (23) as

$$
J_{s+1}\left(\tau, \hat{\boldsymbol{l}}^{\prime}\right)=\frac{\Lambda}{4 \pi} \oint L_{s}\left(\tau, \hat{\boldsymbol{l}}^{\prime}\right) x\left(\hat{\boldsymbol{l}}, \hat{\boldsymbol{l}}^{\prime}\right) d \hat{\boldsymbol{l}}^{\prime} .
$$

The iterative scheme (24)-(27) converges to a 'true' solution. However, if the medium is transparent, the convergence is awfully slow. Also, note, that the at low number of iterations the energy is not conserved.

\subsection{Small-angle approximation}

For sighting angles close to incident angles (i.e. $\mu \approx \mu_{0}$ ), the small angle approximation can be used to compute the transmitted radiance for the case of extended in the forward direction phase functions [28]. The initial boundary value problem is then transformed into

$$
\left\{\begin{array}{c}
\mu_{0} \frac{\partial L(\tau, \hat{\boldsymbol{l}})}{\partial \tau}+L(\tau, \hat{\boldsymbol{l}})=\frac{\Lambda}{4 \pi} \oint L\left(\tau, \hat{\boldsymbol{l}}^{\prime}\right) x\left(\hat{\boldsymbol{l}}, \hat{\boldsymbol{l}}^{\prime}\right) d \hat{\boldsymbol{l}}^{\prime}, \\
\left.L(\tau, \hat{\boldsymbol{l}})\right|_{\tau=0, \mu>0}=\delta\left(\hat{\boldsymbol{l}}-\hat{\boldsymbol{l}}_{0}\right) .
\end{array}\right.
$$

Note that $\mu$ is substituted by $\mu_{0}$ before the derivative. The advantage of the small-angle radiative transfer equation is that it can be solved analytically. In particular, Eq. (28) can be solved by using the spherical harmonics method, i.e. expanding the radiance into Fourier cosine series and then Legendre series. Note that on the left hand side of Eq.(28) we have $\mu_{0} d L / d \tau$ instead of $\mu d L / d \tau$, and thus, all terms in Eq. (28) have the same expansion kernels. The solution reads as follows

$$
L_{\mathrm{SAA}}(\tau, \mu, \varphi)=\frac{1}{4 \pi} \sum_{m=0}^{\infty} \sum_{n=m}^{\infty}\left(2-\delta_{m 0}\right)(2 n+1) \frac{(n-m) !}{(n+m) !} \mathrm{e}^{-\frac{1}{\mu_{0}}\left(1-\Lambda \chi_{n}\right) \tau} \mathrm{P}_{n}^{m}(\mu) \mathrm{P}_{n}^{m}\left(\mu_{0}\right) \cos m \varphi,
$$

where $\delta_{m 0}$ is 1 if $\mathrm{m}=0$ and zero, otherwise, $\chi_{n}$ is the Legendre expansion coefficients of the phase function, while $\mathrm{P}_{n}^{m}$ are the associated Legendre polynomials. The small-angle 
solution gives a Dirac delta function at the upper boundary and a peaked function inside the scattering medium. The amplitude of the peaked function decreases with increasing the optical depth, while its width increases with increasing the optical depth. Fig.2 illustrates the small-angle solution. As we can see, it agrees well with the discrete ordinate solution for angles close to the incident angle.
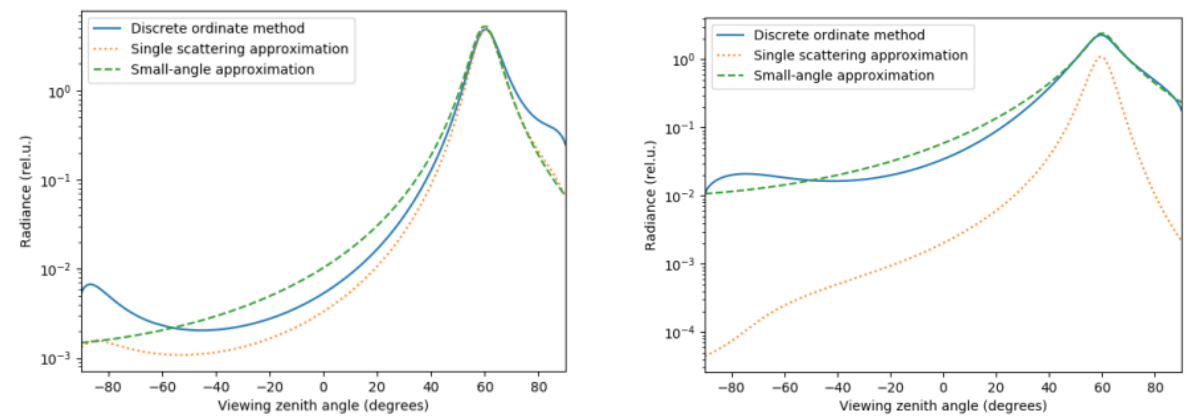

Fig. 2. Comparison between the discrete ordinate solution, the small-angle approximation, and the single scattering approximation. The computations are performed for the Henyey-Greenstein phase function with the asymmetry parameter 0.9 . The single scattering albedo is 0.99 . The angle of incidence is 60 degrees. (left) the optical thickness is 0.3. (right) The optical thickness is 2.0.

\section{Synthetic iterations}

Keeping in mind the advantages and limitations of the exact discrete ordinate approach and approximate solution techniques, we can formulate a hybrid approach based on synthetic iterations [29-30]. Originally such an approach was formulated in nuclear physics. The computations of the radiance field are performed in two steps. At the first step, an approximate solution is found. It should preserve the energy balance (i.e. the integral quantity) while we are not interested in a detailed angular dependency of the radiance field. At the second step, the solution is refined by using the iteration technique similar to Eqs. (25)-(26). However, here at the first iteration we already have a good approximation, while in the original formulation of iterative method, the initial solution based on the single scattering approximation has a large error.

A computationally efficient method of synthetic iterations applied to Eq. (3) consists in the following steps:

1. the anisotropic part of the radiance field (together with the singular component) is expressed by using the small-angle approximation;

2. the remaining regular part of the radiance is computed in the two-stream approximation, which preserves the energy balance;

3. associating the current solution with the source function in Eqs. (25)-(26), the solution 
is refined for given viewing angles by applying iterative scheme (25)-(26). To perform integration over $t$, we make use of expressions for the radiance inside the layer (see Eqs (13)-(15)).

Note that the synthetic iteration method inherits advantages from both approximate and discrete ordinate solution techniques.

\section{Summary}

Analytical discrete transfer theory [9] allowed us to show the relationship of all approximate methods for determining the radiance factor, its accuracy, and applicability. The most significant method for improving the accuracy of approximate solution methods is to isolate the anisotropic part (4) from the solution based on the small-angle approximation (subsection 4.5). The regular part of the solution can be determined by any approximation, which provides an accurate definition of the reflection coefficient. To improve determining the accuracy of the angular distribution of the radiance factor (BRDF), the method of synthetic iterations (section 5) is the most effective. It seems that this approach solves all the problems of determining the radiance factor of the real-world objects.

\section{References}

1. Kurt, M., Edwards, D.A.: Survey of BRDF models for computer graphics. Computer Graphics (ACM) 43(2), 4 (2009)

2. Lai, Q., Liu, B., Zhao, J., Zhao, Z., Tan, J.: BRDF characteristics of different textured fabrics in visible and near-infrared band. Optics Express, 28(3), P.3561 (2020)

3. The optics of Ibn Al-Haytham. Books I-III: On Direct Vision. Translated with introduction and commentary by A.I. Sabra. The Warburg institute university of London, London (1989)

4. Fabry, C.: Introduction Générale à la Photométrie, Editions de la revue d'optique théoretique et instrumentale. Paris (1927).

5. ILV: International lighting vocabulary, CIE S 017/E:2011. Commission Internationale de L'Eclairage, Vienna (2020)

6. Pokrowski, G.I.: Zur Theorie der diffusen Lichtreflexioni. Zeit. f. Physik, 30, 66 (1924)

7. Torrance, K.E., Sparrow, E.M.: Theory for off-specular reflection from roughened surfaces. JOSA, 57(9), 1105-1114 (1967).

8. Basov, A.Y., Budak, V.P.: Mathematical model of a surface radiance factor. CEUR Workshop Proceedings, 29th International Conference on Computer Graphics and Vision, GraphiCon 2019; Bryansk; Russia; 23 - 26 September 2019, 2485, 43-47 (2019)

9. Afanas'ev, V.P., Basov, A.Y., Budak, V.P., Efremenko, D.S., Kokhanovsky, A.A.: Analysis of the discrete theory of radiative transfer in the coupled "ocean-atmosphere" system: current status, problems and development prospects. Journal of Marine Science and Engineering, 8(3), 202 (2020). https://doi.org/10.3390/jmse8030202

10. Chandrasekhar, S.: Radiative transfer. Dover publications, inc. New York (1950)

11. Milne, E.A: The reflection effect of the eclipse binaries. Mon. Not. Roy. Astrophys. Soc., LXXXVII, 43-49 (1926). 
12. Bohren, C.F., Huffman, D.R.: Absorption and Scattering of Light by Small Particles. Wiley (Apr 1998). https://doi.org/10.1002/9783527618156

13. Budak, V.P., Klyuykov, D., Korkin, S.V.: Complete matrix solution of radiative transfer equation for pile of horizontally homogeneous slabs. J Quant Spectrosc Radiat Transfer 112(7), 1141-1148 (2011). https://doi.org/10.1016/j.jqsrt.2010.08.028

14. Budak, V.P., Efremenko, D.S.; Shagalov, O.V.: Efficiency of algorithm for solution of vector radiative transfer equation in turbid medium slab. J. Phys. Conf. Ser. 369, 012021 (2012). https://doi.org/1088/1742-6596/369/1/012021

15. Karp, A.H.; Greenstadt, J.; Fillmore, J.A.: Radiative transfer through an arbitrarily thick, scattering atmosphere. J. Quant. Spectrosc. Radiat. Transf. 24, 391-406 (1980). doi: 10.1016/0022-4073(80)90074-6

16. Efremenko, D.S., Molina García, V., Gimeno García, S, Doicu, A.: A review of the matrixexponential formalism in radiative transfer. Journal of Quantitative Spectroscopy and Radiative Transfer 196, 17-45 (2017). https://doi.org/10.1016/j.jqsrt.2017.02.015

17. Plass, G.N., Kattawar, G.W., Catchings, F.E.: Matrix Operator Theory of Radiative Transfer. Appl. Opt. 12, 314-326 (1973)

18. Kolmogorov, A.N.: Combinatorial foundations of information theory and the calculus of $\begin{array}{llllll}\text { probabilities. } & \text { Russ. } & \text { Math. } & \text { Surv. } & \text { 38, }\end{array}$ https://doi.org/10.1070/RM1983v038n04ABEH004203

19. Budak, V.P., Shagalov, O.V., Zheltov, V.S.: Numerical radiative transfer modeling in turbid medium slab. Proc. of SPIE, 9292, 92920Y (2014).

20. Schuster, A.: The influence of radiation of the transmission of heat. Phil.Mag., 5, 243-257 (1903)

21. Schwarzschild, K.: Über das Gleichgewicht der Sonnenatmosphäre. Nachr. Konig. Gesel. der Wiss., Gottingen, 1,41-53 (1906).

22. Davidson, B.: Neutron Transport Theory. Clarendon, Oxford, UK, 1957

23. Ambartsumian, V.A.: On the problem of diffuse reflection of light. J. Phys. USSR, 8, 65-75 (1944)

24. Flatau, P.J., Stephens, G.L.: On the Fundamental Solution of the Radiative Transfer Equation. J. Geophys. Res., 93, 11037-11050 (1988)

25. Chwolson, O.D.: Grundzüge einer matimatischen Theorie der inneren Diffusion des Licht. Bull. Acad. Imp. Sci. St. Petersbourg. 33, 221-256 (1889)

26. Lommel, E.: Die Photometric der diffusen Zuruckwerfung. Sitzber. Acad. Wissensch. Munchen, 17, 95-124 (1887)

27. Lenoble ,J., Herman, M., Deuzé, J.L., Lafrance, B., Santer, R., Tanré, D.: A successive order of scattering code for solving the vector equation of transfer in the earths atmosphere with aerosols. Journal of Quantitative Spectroscopy and Radiative Transfer, 107(3), 479-507 (2007)

28. Kokhanovsky, A.A.: Small-angle approximations of the radiative transfer theory. Journal of Physics D: Applied Physics, 30(20), 2837-2840 (1997)

29. Budak, V.P., Kaloshin, G.A., Shagalov, O.V.; Zheltov, V.S.: Numerical modeling of the radiative transfer in a turbid medium using the synthetic iteration. Opt. Exp. 23, A829-A840 (2015). https://doi.org/10.1364/OE.23.00A829

30. Budak, V.P., Zheltov, V.S., Lubenchenko, A.V., Freidlin, K.S., Shagalov, O.V.: A fast and accurate synthetic iteration-based algorithm for numerical simulation of radiative transfer in a turbid medium. Atmos. Ocean. Opt. 30, 70-78 (2017). 